\title{
Strong thermostable interference coatings for IR optical elements
}

\author{
Yu.A.Zagoruiko, O.A.Fedorenko, N.O.Kovalenko, P.V.Mateychenko \\ Department of Optical and Constructional Crystals STC "Institute for Single Crystals", NAS of Ukraine, \\ Lenin Ave, 60, Kharkov 310001, Ukraine
}

\begin{abstract}
Interference $\mathrm{ZnO}$ coatings for IR optical elements are obtained by the method of photothermal oxidation on the surface of crystalline ZnSe. Investigated are their mechanical and optical properties. The obtained oxide coatings possess high adhesion, homogeneity, withstand repeated thermocycling and irradiation with high-intensity $\mathrm{CO}_{2}$-laser radiation. Revealed is the formation of islets of oxide films with elevated mechanical strength.
\end{abstract}

Keywords: $\mathrm{ZnSe}$, antireflection $\mathrm{ZnO}$ coatings, adhesion, mechanical strength.

Paper received 04.10.99; revised manuscript received 14.11.99; accepted for publication 21.03.00.

\section{Introduction}

Experimental investigations aimed at search for new strong thermostable antireflection coatings suitable for a wide spectral range, are extremely topical for the creation of modern devices meant for the IR region. In particular, such a work is necessary for making IR optical elements which can operate within a wide temperature range and at high-intensity optical radiation [1-4]. Especially significant these investigations are for the development and creation of thermostable multifunctional optical elements transparent both in the visible and in the IR regions [5].

Known types of interference coatings for IR optical elements made on the base of crystalline wide-gap $\mathrm{A}^{2} \mathrm{~B}^{6}$ materials provide a good antireflection, however, their mechanical strength and thermal stability are not high enough. Moreover, the process of covering the optical elements with such coatings is rather expensive and labour-consuming.

To solve the problem in question, an original method of photothermal antireflection of $\mathrm{ZnSe}$ optical crystalline elements has been proposed [1]. The use of this method provides the formation of strong interference $\mathrm{ZnO}$ coatings characterized by a high adhesiveness, on the surface of the optical elements to be utilized within a wide spectral range.

\section{Experimental procedure}

The following two methods of oxidation were used: thermal oxidation (TO) in darkness and thermal oxidation at simultaneous action of optical irradiation, i.e. photothermal oxidation (PTO). In these experiments optical radiation sources with $0.2-0.7 \mu \mathrm{m}$ wavelengths (mercury lamps), were used. The technological processes of TO and PTO were realized within $630 \ldots 800 \mathrm{~K}$ temperature range. The duration of TO and PTO processes was $1.8 \cdot 10^{2} \div 2.2 \cdot 10^{4} \mathrm{~s}$. The investigations were carried out on polished $\mathrm{ZnSe}$ samples oriented in (111) crystallo-graphic plane.

The coefficients of optical absorption of IR optical elements were measured by the method of adiabatic laser calorimetry at the working wavelength of $\mathrm{CO}_{2}$-lasers $(\lambda=10.6 \mu \mathrm{m})$. The temperature dependences of the absorption coefficient $\beta$ were determined within $285 \div 750 \mathrm{~K}$ temperature interval.

The mechanical strength of the optical coatings was investigated by the methods of microhardness and sclerometry by means of a PMT-3 device using Vickers diamond pyramid.

The oxide coatings were studied by the method of scanning electron microscopy combined with X-ray microanalysis using a JSM-820 electron microscope. 


\section{Yu.A. Zagoruiko et al.: Strong thermostable interference coatings for IR optical elements}

\section{Results}

Based on the investigations of the processes of thermal [3] and photothermal [5] oxidation of the crystalline zinc selenide, the authors developed a method of obtaining photostimulated antireflection coating on IR optical elements made from $\mathrm{ZnSe}[4,6]$. Performed were comparative investigations of the optical, structural and thermomechanical characteristics of $\mathrm{ZnSe}$ optical elements with $\mathrm{ZnO}$ interference coatings produced by the thermal and photothermal methods.

The interference oxide coatings prepared by photostimulated oxidation were found to have lower values of optical absorption at $\lambda=10.6 \mu \mathrm{m}$, higher adhesion to the surface of the crystalline substrate, better light transmission in the spectral range of $2.5 \ldots 12.0 \mu \mathrm{m}$. These results testify to a more perfect structure of $\mathrm{ZnO}$ coatings prepared by the method $[4,6]$. This conclusion is also confirmed by the study of the phase composition and structure of oxide layers carried out by the method of X-ray diffraction analysis. Thus, the obtained data show that photostimulated oxidation allows to obtain highly textured $\mathrm{ZnO}$ films [7]. The direction [0001] of the textured $\mathrm{ZnO}$ films coincides with the normal to the surface of the sample (crystallographic direction [111] of $\mathrm{ZnSe}$ single crystal).

The method of adiabatic laser calorimetry at $\lambda=10.6 \mu \mathrm{m}$ was used to determine the coefficients of total absorption $\left(\beta_{\Sigma}\right)$ for $\mathrm{ZnO}-\mathrm{ZnSe}-\mathrm{ZnO}$ heterostructures produced by the methods of TO and PTO. These investigations showed that for the heterostructures with the transmission coefficients higher than $95 \%(\lambda=10.6 \mu \mathrm{m})$, the value of $\beta_{\Sigma}$ is approximately by $15 \ldots 20 \%$ larger than the initial value of optical absorption of $\mathrm{ZnSe}$ crystalline substrates.

The temperature dependences of the total optical absorption coefficient $\beta_{\Sigma}$ of the obtained heterostructures (with $\mathrm{ZnO}$ layer thickness $\leq 3 \mu \mathrm{m}$ ), do not essentially differ from those of uncoated $\mathrm{ZnSe}$ samples [8]. Experimental investigations showed that within $0 \ldots 100^{\circ} \mathrm{C}$ temperature range the optical characteristics of $\mathrm{ZnO}-\mathrm{ZnSe}$ heterostructures slightly vary both in the visible and the IR region of the spectrum. The optical elements with antireflection $\mathrm{ZnO}$ coatings are characterized by thermal stability and mechanical strength. They withstand repeated thermocycling $(280 \mathrm{~K} \rightarrow 700 \mathrm{~K} \rightarrow 280 \mathrm{~K}$, the rate of temperature change $\geq 5 \mathrm{~K} / \mathrm{sec}$ ) and long-duration (not shorter than $15 \mathrm{~min}$ ) laser irradiation with an intensity up to 10 $\mathrm{kW} / \mathrm{cm}^{2}$ (for $\lambda=10.6 \mu \mathrm{m}$ and the irradiation area with a diameter of $1 \mathrm{~mm}$ ) at temperatures up to $610 \mathrm{~K}$. This fact allows to assume that $\mathrm{ZnO}$ coatings may be used as thermostable interference antireflection coatings for $\mathrm{ZnSe}$ crystalline optical elements working in a wide range of the spectrum.

The mechanical strength of the optical coatings was investigated by the methods of microhardness and sclerometry on a PMT-3 device using the diamond Vickers pyramid. The value of microhardness was measured by the conventional method with indentor loads $(P)$ of $100 \div 2 \mathrm{~g}$. Investigated were the closely packed faces with oxide coatings obtained under UV-irradiation.
The performed microhardness investigation shows that at all the values of load the indentation has clear edges, and the film does not peel off the surface. This testifies to a strong adhesion of the film to the sample surface. Radial cracks formed around the impression are similar to those caused by indenting the surface of $\mathrm{ZnSe}$ single crystals without oxide films.

The results of scratching investigation show that the film adhesion to the crystal surface is good, since at all the loads no peeling of the film is observed. At $50 \div 10 \mathrm{~g}$ loads scratches are brittle. Starting from loads of $5 \mathrm{~g}$ and lower, brittle failure around the scratches is absent. On the faces (111) and (111) the scratch width at $P=5 \mathrm{~g}$ is 6.7 and 6.2 , respectively. At $P=0.5 \mathrm{~g}$ no scratching trace is observed on both faces.

Revealed on the (111) plane were oxide film islets with a thickness larger than that of the surrounding surface. They were shaped as ovals with dimensions of the order of several tenth parts of millimeter. Such islets had higher hardness and cracking resistance in comparison with the surrounding surface. In particular, within the islets the indentations were clear in shape even at $P=100 \mathrm{~g}$, and brittle halos around them were absent. At $P=5 \mathrm{~g}$ the value of microhardness within the islets ran into $113 \mathrm{~kg} / \mathrm{mm}^{2}$, whereas beyond the islet boundaries such a value was equal to $74 \mathrm{~kg} / \mathrm{mm}^{2}$. The results of scratching testify that the islets of oxide films had higher cracking resistance, too. Fig. 1 presents such an islet on the surface of $\mathrm{ZnSe}$ single crystal with a crack obtained at a load of $10 \mathrm{~g}$. As is seen, around the segment of the crack beyond the islet boundaries brittle damage takes place; inside the islet such a damage is absent.

The islets obtained in the process of oxidation were studied by the method of scanning electron microscopy combined with X-ray microanalysis using a JSM-820 electron microscope. Shown in Fig. 2 is the image of such an islet obtained in the regime of reflected electrons (COMPO), as well as X-ray maps of element distribution in this area. In the regime of reflected electrons the contrast of image is determined by the average atomic number of the target. As is seen, the region of the islet is darker than the surrounding matrix, therefore its average atomic number is lower. Seen in the figure is an indentor scratch passing over the islet. Bright white grains along the scratch (beyond the islet) correspond to ZnSe grains which have reached the surface as a result of brittle failure of the oxide film outside the islet. The segment of the scratch passing over the islet has no such grains.

Presented in the maps of element distribution studied in X-rays (O $K_{\alpha}, \mathrm{Zn} K_{\alpha}$ and Se $K_{\alpha}$ lines $)$ is the same microregion of the sample surface. Bright white grains along the scratch in the map built in $\mathrm{Se} K_{\alpha}$ line radiation correspond to the dark areas in the $\mathrm{O} K_{\alpha}$ and $\mathrm{Zn} K_{\alpha}$ maps, and are treated as $\mathrm{ZnSe}$ grains on the oxide film background. The islet manifests itself with the greatest contrast in the Se $K_{\alpha}$ map (in the form of a dark area). In the $\mathrm{Zn} K_{\alpha}$ map it is weakly seen as a light area (since the weight concentration of $\mathrm{Zn}$ in $\mathrm{ZnSe}$ is lower in comparison with that in $\mathrm{ZnO}$ ). In the $\mathrm{O} K_{\alpha}$ map the islet is not 


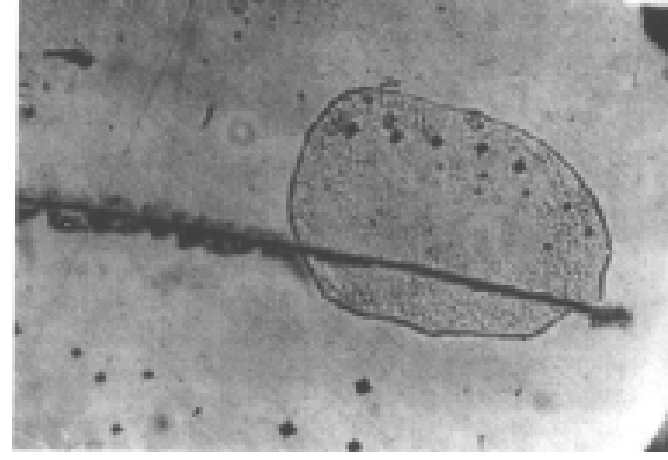

a)

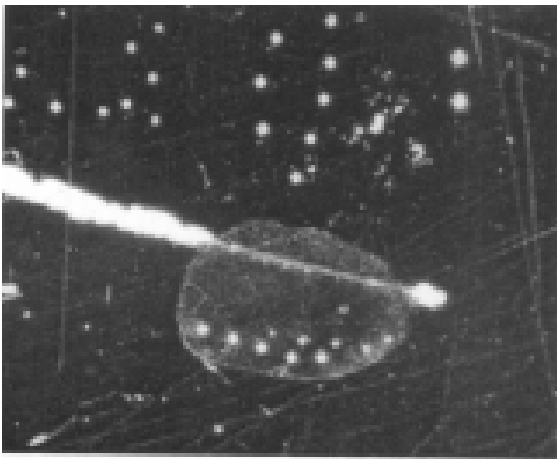

b)

Fig. 1. "Islet" film on (111) surface of ZnSe single crystal: a) in light field; b) in dark field.

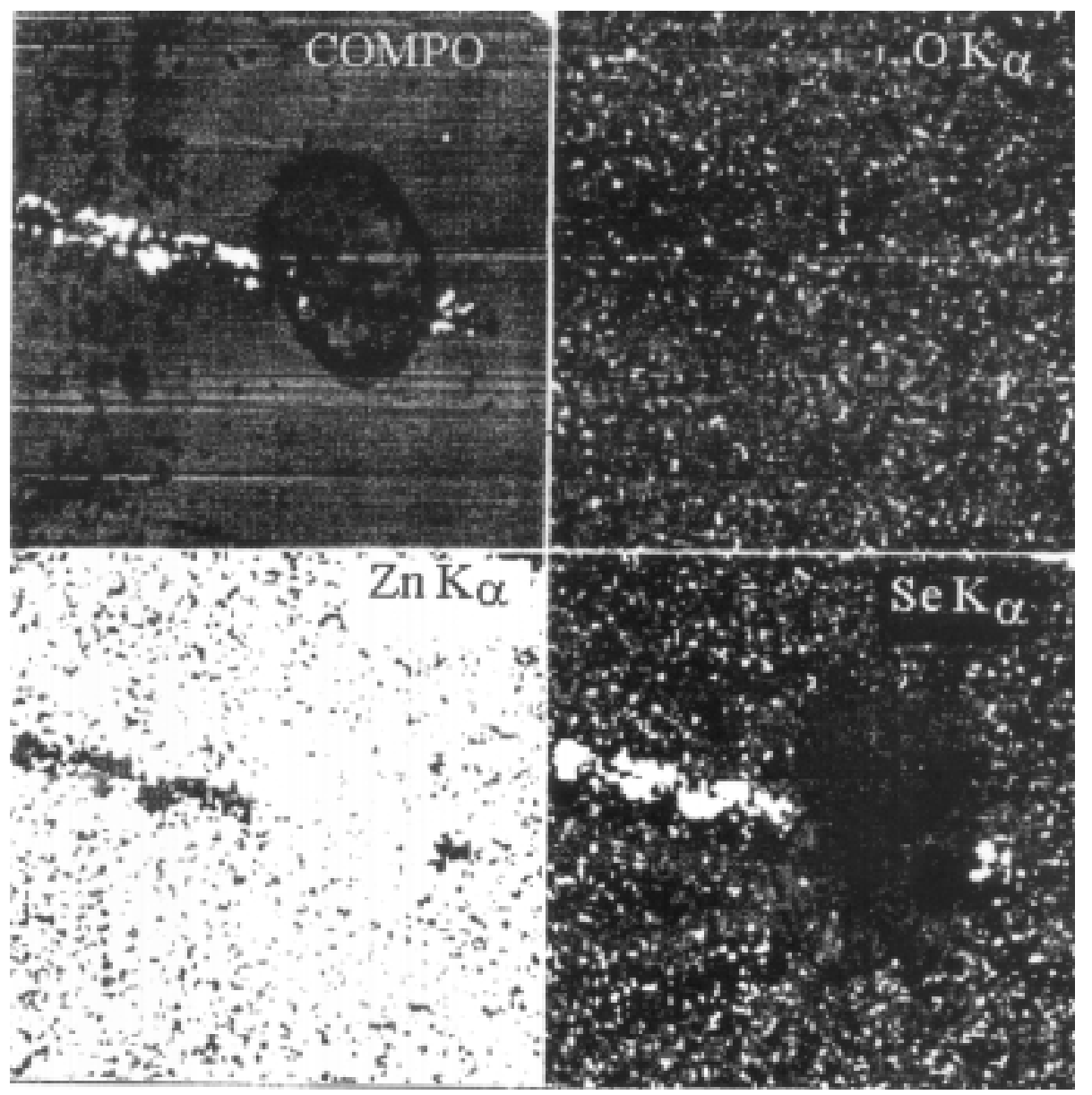

Fig. 2. X-ray maps of element distribution in the vicinity of «islet» films: COMPO- the image of oxide film "islet" obtained in COMPO regime; $\mathrm{OK}_{\alpha}$-distribution of $\mathrm{O} ; \mathrm{ZnK}_{\alpha}$-distribution of $\mathrm{Zn}$; $\mathrm{SeK}_{\alpha}$-distribution of Se.

visible at all. Such a picture of contrasts in the X-ray maps may be explained by different depths of X-ray exit out of the sample depending on the energy of the given line. "Soft" $\mathrm{O} K_{\alpha}$ radiation $\left(E_{c}=0.52 \mathrm{eV}\right)$ reaches the surface without essential attenuation and out of smaller depths in comparison with "hard" Se $K_{\alpha}$ radiation $\left(E_{c}=11.2 \mathrm{keV}\right)$. In the $\mathrm{O} K_{a}$ map contrast in the region of the islet is absent due to the fact that the whole of the surface (with the exception of the grains located along the scratch) is covered by a rather thick oxide layer. In the $\mathrm{Zn} K_{\alpha}\left(\mathrm{E}_{\mathrm{c}}=8.6 \mathrm{keV}\right)$ and Se $K_{\alpha}\left(E_{c}=11.2 \mathrm{keV}\right)$ maps contrast is seen. This is explained by the fact that the radiation of these lines has a higher penetration ability, and reaches the surface out of larger depths in comparison with the thickness of the 
Yu.A. Zagoruiko et al.: Strong thermostable interference coatings for IR optical elements

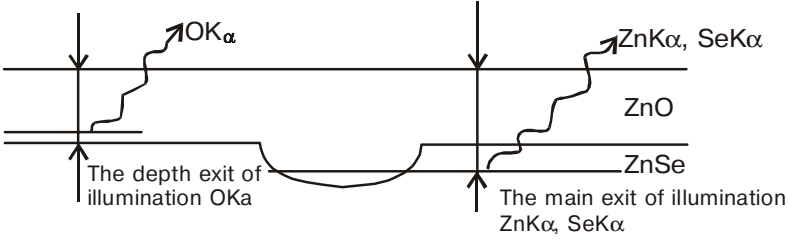

Fig. 3. Schematic structure of oxide layer in the vicinity of «islet» films.

oxide film surrounding the islet. The structure of the oxide layer is schematically shown in Fig.3.

It should be noted that the interference oxide films are resistant to organic solvents.

\section{Conclusions}

As it is ascertained experimentally, the highest thermostability is characteristic of the elements with $\mathrm{ZnO}$ coatings obtained by the method of photostimulated oxidation.

Such $\mathrm{ZnO}$ coatings are found to possess an elevated degree of adhesion to the surface of crystalline $\mathrm{ZnSe}$. On (111) plane of $\mathrm{ZnSe}$ crystalline substrates, revealed are islets of oxide films with a thickness larger than that of the surrounding surface. Such islets are characterized by higher hardness and cracking resistance in comparison with the surrounding surface.

Experimentally verified is the possibility to use $\mathrm{ZnO}$ coatings as strong homogeneous thermostable interference antireflection coatings for $\mathrm{ZnSe}$ crystalline optical elements meant for a wide region of the spectrum.

\section{Acknowlegement}

This work is partly supported by Science and Technology Center in Ukraine (STCU), Project \# 380.

\section{References}

1. K.Tomura, H.Ohnishi, M.Yoshisawa, ZnO films formed by oxidation of ZnSe films // Jap. J. Appl. Phys., 13, pp. 939-943 (1974).

2. I.P.Kuz'mina, V.A.Nikitenko. Zinc oxide. Obtaining and optical properties. Moscow, «Nauka» 1984.

3. M.P.Kulakov, A.V.Fadeev, Oxidation of mechanically polished zinc selenide at heating in air // Izv. AN SSSR. Neorg. Mater, 19, №3, pp. 347-351 (1983).

4. Yu.A.Zagoruiko. ZnO Films Obtained on ZnSe Single Crystals Surfaces by Photothermal Oxidation Method and their properties. EMRS 1993 Fall Meeting $4^{\text {th }}$ European East-West Conference@ Exhibition on Materials and Processes. StPetersburg (Russia), October 17-21, 1993.

5. Yu.A.Zagoruiko, O.A.Fedorenko, N.O.Kovalenko. Thermostable oxide coatings for IR optical elements based on crystalline zinc selenide. E-MRS 1999 Spring Meeting, Symposium I "Microcrystalline and Nanocrystalline Semiconductors", p.I-54, Strasbourg, France.

6. Yu.A.Zagoruiko, V.K.Komar, V.Yu. Rastorgueva, V.N.Krivoshein. A Method of making Antireflecting Optical Elements of Zinc Selenide. Patent of Russian Federation № $1349543,1993$.

7. Yu.A.Zagoruiko, O.A.Fedorenko. Peculiarities of Laser Damage of $A^{2} B^{6}$. Single Crystals.1997, Proceedings of $29^{\text {th }}$ annual Boulder damage symposium, SPIE, vol. 3244, pp. 650655 (1997).

8. Yu.A.Zagoruiko, O.A.Fedorenko. Temperature Dependences of IR-Absorption and $\mathrm{CO}_{2}$ - laser Damage of $\mathrm{ZnSe}$ and CdS Single Crystals. Abstracts of 30 th Annual Boulder Damage Symposium., Boulder, USA (1998). 